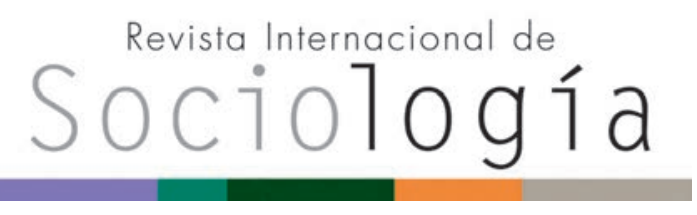

Revista Internacional de Sociología RIS vol. 75 (3), e073, julio-septiembre, 2017, ISSN-L:0034-9712 doi: http://dx.doi.org/10.3989/ris.2017.75.3.15.171

\title{
LOS ESTILOS DE AMOR EN ESTUDIANTES UNIVERSITARIOS. Diferencias en función del sexo-género
}

\author{
JAVIER RODRÍGUEZ-SANTERO \\ Universidad de Sevilla, España. \\ jarosa@us.es \\ ORCID iD: http://orcid.org/0000-0001-5727-4545
}

\author{
M. a Ángeles García-CARPInTERo Muñoz \\ Universidad de Sevilla, España. \\ agcarpin@us.es \\ ORCID iD: http://orcid.org/0000-0003-4961-484X
}

\author{
ANA MARÍA Porcel GÁlVEZ \\ Universidad de Sevilla, España. \\ aporcel@us.es \\ ORCID iD: http://orcid.org/0000-0001-8461-6379
}

Cómo citar este artículo / Citation: RodríguezSantero, J.; M. A. García-Carpintero Muñoz y A. M. Porcel Gálvez. 2017. "Los estilos de amor en estudiantes universitarios. Diferencias en función del sexo-género". Revista Internacional de Sociología 75 (3): e073. doi: http://dx.doi.org/10.3989/ris.2017.75.3.15.171

\section{RESUMEN \\ En este trabajo describimos los estilos de amor que se dan entre la población juvenil de la Universidad de Sevilla y hacemos un análisis de las diferencias según el sistema sexo-género. Para ello, se ha realizado un estudio descriptivo y transversal que ha empleado una adaptación contextualizada de la escala Love Attitudes Style (Hendrick et al. 1998). Este instrumento validado de recogida de datos, que hemos denominado 'ReLAS', fue administrado finalmente a 447 estudiantes de la Universidad de Sevilla (267 mujeres y 180 hombres). Los resultados señalan que, por lo general, los sujetos cuen- tan con un concepto idealizado y romántico del amor, en el que la sexualidad y los aspectos más pasionales o de atracción son secundarios, aunque con ciertos matices importantes, como hemos podido comprobar al estudiar las diferencias por sexo atribuibles a los roles de género.}

Palabras Clave

Relaciones de pareja; Universidad.
LOVE ATTITUDES STYLES

AMONGST COLLEGE STUDENTS. Differences by sex-gender
Copyright: (C) 2017 CSIC. Este es un artículo de acceso abierto distribuido bajo los términos de la licencia Creative Commons Attribution (CC BY) España 3.0.

Recibido: 21/10/2015. Aceptado: 21/09/2016.

\section{ABstract}

This paper aims at describing love attitudes styles amongst the youth at Universidad de Sevilla and reviews differences in such attitudes by sex-gender system. To do so, a descriptive and interdisciplinary study was conducted using a contextualized adaptation of the Love Attitudes Style Scale (Hendrick et al. 1998). This validated data collection tool, referred to as 'ReLAS,' was applied to 447 students from the said Spanish university (267 women and 180 men). Our findings indicate that, overall, the subjects possess an idealized, romanticized view of love, whereby factors such as sexuality, passion and attraction play a secondary if significantly nuanced role, as emerged when looking into sex-based differences that are attributable to gender roles.
KEYWORDS

Relationships; University. 


\section{INTRODUCCIÓN}

El amor es un fenómeno universal, polisémico, dinámico, inmerso en un tiempo y en un constructo sociocultural determinado, cuya imagen y significado varía de unos individuos a otros en función de la etapa vital, las personas con las que se relacionan y la evolución de la relación (Hendrick et al. 1988; Ubillos et al. 2001; Sangrador 1993; Esteban y Távora 2008). Desde principios del siglo XIX, en las sociedades occidentales se vincula el amor romántico, la sexualidad y el matrimonio, resultando un tema de interés por su importancia como eje vertebrador de la estructura social (Sternberg 1986 y 1996; Walker y Barton 1988; Navarro 2006). Es un tema interesante de análisis, ya que a través de la sexualidad y de los vínculos que se establecen en las parejas podríamos explicar las relaciones simétricas y asimétricas que operan entre los sexos, y que van más allá del amor como emoción individual. Esta relación biunívoca entre el individuo y la cultura donde está inmerso ha sido explicada tanto desde una perspectiva culturalista, con base psicológica y antropológica (Illouz 2012), como desde la sociobiológica, con bases biologicistas y evolucionistas, que entiende la funcionalidad del amor como necesidad de continuidad de la especie a través de la procreación (Fisher 1992 y 2005). En nuestra investigación nos situamos en la perspectiva culturalista, aunque describiremos también las tesis sociobiológicas.

Los planteamientos sociobiológicos, como el de la antropóloga y bióloga Hellen Fisher (2005), explican que el amor y sus manifestaciones (deseo sexual, romance y apego hacia la pareja) son fenómenos universales, independientes de la edad, el sexo o la raza. Concretamente, la autora alude a tres circuitos cerebrales: el impulso sexual que tiene la función de elegir a la mejor pareja disponible, el amor romántico que tiene función de focalizar la atención y emociones en esa pareja, y, por último, el apego o cariño, que tiene la función de establecer lazos para criar a los hijos. Esta situación se mantiene durante el periodo de procreación y crianza, que duraría según la autora aproximadamente cuatro años. Esta interrelación de los tres sistemas cerebrales sería tan antigua como la evolución del hombre como especie y asegura la perpetuación de la misma.

La sexualidad humana se articula así como el cruce indiscutible entre naturaleza y cultura, ya que a través del vínculo sexual se establecen los lazos estructurales del parentesco. Esta estructura, definida antaño por Lévi-Strauss, tiene un amplio abanico diferencial entre unas sociedades y otras, pero subyace en todas ellas un elemento común y universal, el control sobre la sexualidad femenina y la dominación de los varones (Rubin 2011). El materialismo histórico de Engels ([1884] 2008) sitúa el control del varón sobre la sexualidad femenina en el nacimiento de la propiedad privada y la familia patriarcal, dada la necesidad de generar herederos de paternidad indiscutible.

La larga sombra que proyecta este patriarcado milenario sigue hoy latente en las sociedades postindustriales formalmente igualitarias. Anne Jónasdóttir (1993) afirma que el patriarcado actual se basa en la explotación del poder productivo del amor, pues es un proceso social específico o sistémico que organiza la capacidad sensual y la fuerza creativa del amor humano. En la misma línea, nos resultan interesantes las ideas de Illouz (2007; 2009; 2012) acerca de cómo el capitalismo determina el amor, dando lugar a lo que ella denomina "capitalismo emocional", en el que todo se resume en el coste-beneficio, prima la ausencia de compromiso, el desamor se convierte el algo cada vez más usual, y gana terreno el "capital sexual". Todo ello considerablemente favorecido por las nuevas redes sociales que se forjan a través de Internet y potencian esa ley de la oferta y la demanda emocional.

Según Castells y Subirats (2007), los mandatos específicos que deben cumplir los individuos para ser verdaderos 'hombres' o 'mujeres', al haber sido interiorizado desde la niñez, pasan a formar parte de nuestros gustos y deseos. Desde la infancia, la niña y el niño se socializan en una cultura que les inculca los roles de género respecto al amor (Barrón et al. 1999), mediante un conjunto complejo de producción cultural que comprende los cuentos infantiles, canciones, películas o novelas; generalmente en forma de relatos saturados de estereotipos, que recrean el amor heterosexual entre un hombre y una mujer (Ferrer, Bosch y Navarro 2010; Montañés et al. 2015). Se les va adoctrinando así, sobre el proceso de elección de pareja, o lo que es lo mismo, sobre el cómo, cuándo y de quién debe uno o una enamorarse (Moreno y Sastre 2010; Padilla y Díaz-Loving 2013). Estas disposiciones internalizadas constituyen para Illouz el "habitus" y son responsables de que los individuos busquen, de manera no consciente, parejas de similar estatus social, económico y cultural, a pesar de las declaraciones explícitas en las que aluden a la irrelevancia de tales aspectos. Las culturas aprueban o desaprueban ciertos comportamientos relacionados con el amor según el lugar, el momento en el que se producen y el sexo de quien las vive. En la socialización de género, a la mujer se le ha asignado tradicionalmente el mundo de los afectos y las emociones, igual que al hombre el poder del pensamiento racional. De ahí el interés por analizar el amor, ya que formaría parte de la estructura del sistema sexo-género (Ferrer, Bosch y Navarro 2010; Montañés et al. 2015).

Existen diferentes creencias sobre el amor que dependen de los recursos sociales, la demografía, el estatus y, sobre todo, de las jerarquías de poder y género. Aunque todo parece apuntar a que a mayor igualdad social y económica entre sexos, mayores semejanzas en los comportamientos de hombres y mujeres con respecto al amor (Eagly y Wood 1999; 
Ajenjo y García 2014). En este sentido, Neto (2012) afirma que el desarrollo socio-económico refuerza el individualismo e incide directamente en que, tanto hombres como mujeres, otorguen gran importancia al amor pasional.

Según la teoría en la que nos posicionemos nos encontraremos con diferentes enfoques sobre el amor. A principios del siglo XX, Scheler (1996) reflexiona sobre la fenomenología de los afectos como parte esencial para explicar la naturaleza humana y le otorga gran importancia a los sentimientos de simpatía y amor, a la hora de establecer relaciones con los demás. Explica la simpatía como el fundamento de toda relación interpersonal, que facilita una comprensión genuina de las personas y crea una comunidad de sentimientos. Sin embargo, el amor da un paso más, supera esos límites y tiene la capacidad de establecer una relación profunda valorando la autonomía y diversidad del otro. Esto explicaría que mientras elegimos a los amigos por afinidad y las compensaciones gratificantes que esa relación nos aporta, el amor tiene un elemento añadido de irracionalidad que supone la continuidad del vínculo a pesar de que la relación no sea gratificante (Scheler 1996; Adrián 2007).

Desde la psicología, a partir de los años 70 , se hacen esfuerzos considerables por conceptualizar el amor y categorizarlo. Sternberg (1986) propone una teoría triangular del amor y habla de tres elementos básicos: la pasión, la afinidad y el compromiso, que generan distinto tipo de relación según sea la interrelación entre ellos. Los tres elementos están presentes en las distintas fases temporales del amor, aunque con distinta intensidad. Primero aparecería la pasión, que va disminuyendo con el tiempo a la vez que se van afianzando la afinidad y el compromiso. Para dicho autor, el amor es un compromiso incondicional y protector entre dos partes que contribuyen mutuamente a su bienestar y desarrollo. Para Sternberg (1986) el amor es realmente una historia, y los personajes sus autores.

La teoría del amor de Sangrador (1993) alude a tres componentes: actitud, emoción y conducta. Pudiendo entenderse como una atracción o actitud positiva hacia otra persona, una emoción que incluye ciertas reacciones fisiológicas, o una conducta que llevaría a cuidar y atender las necesidades del otro. Para Petersen y Thompson (2005), es una extensión de fronteras del ego hacia alguien que nos gusta en nuestra vida e identidad.

En Colors of love theory, Lee (1973) hace una taxonomía de los distintos estilos de amor distinguiendo tres primarios (Eros, Ludus, Storge) y tres secundarios (Manía, Pragma, Ágape). Eros es el amor pasional, el enamoramiento a primera vista que supone una atracción intensa por la pareja, no solo física sino también emocional, son amantes con alta autoestima y confianza en el otro. Ludus o amor placentero, supone una relación sin implicación emocional, sin celos ni expectativas futuras, con una sexualidad permisiva y abierta donde las reglas del juego están claras y pueden existir más socios sexuales. Storge es el amor que nace de la amistad y el afecto, se buscan similitudes y afinidades en las posibles parejas para entablar una relación armónica basada en el cariño. No valoran tanto la apariencia física o la satisfacción sexual, porque lo que se busca en la relación es un compromiso a largo plazo no un apasionamiento a corto plazo. Manía es el amor obsesivo, adictivo, celoso, posesivo, desconfiado, marcado por grandes conflictos en la pareja a causa de la invasión de los espacios individuales. Pragma se refiere a la búsqueda práctica de intereses en la pareja adecuada, con compatibilidad no solo física sino también, y sobre todo, de intereses. Ágape es el amor altruista, de renuncia personal y entrega desinteresada, donde las necesidades de la pareja están priorizadas sobre las propias.

Estos estilos de amor no permanecen inalterables, más bien varían a lo largo de toda la vida según factores internos y externos al individuo. La forma de vivir y las expectativas de los distintos tipos de amor condicionan las relaciones de pareja, considerándose esta como una estructura que se establece entre dos personas que intercambian en el tiempo emociones, satisfacción sexual y apoyo psicoafectivo, con distinta duración e intensidad. Esa relación puede ser simétrica o asimétrica, según lo experimente cada miembro de la misma (Barbosa et al. 2011).

C. Hendrick y S. Hendrick (1986) desarrollan una escala de medida para dichos estilos de amor, reducida posteriormente para facilitar su administración (Hendrick et al. 1998), denominada Love Attitudes Scale (LAS), donde se exploran elementos individuales (emocionales y rasgos de personalidad) relacionales (sociales, culturales y familiares) y otros asociados al sistema de valores y creencias. Entendemos los distintos tipos de amor como una temática objeto de estudio justificada por la relación determinante entre las relaciones de pareja y la estructura del sistema sexo-género. El deseo de conocer en mayor profundidad este fenómeno nos obliga a contar con instrumentos estandarizados que permitan evaluar con validez y fiabilidad este constructo (Rodríguez-Castro et al. 2013). Por ello, aunque existe abundante literatura sobre el amor en la edad adulta (Ubillos et al. 2001; Esteban y Távora 2008; Ferrer et al. 2008; Ferrer et al. 2010), hemos adaptado la escala LAS al ámbito universitario, con objeto de conocer el tipo de relaciones amorosas que considera la población juvenil universitaria, y analizar si existen diferencias de género en sus planteamientos. Este estudio resulta interesante porque nos permitiría conocer la idea que subyace tras el amor romántico (tipo de pareja, dinámicas dentro de 
la misma, condicionantes...) y detectar elementos de socialización de género.

\section{Metodología}

Estudio descriptivo mediante encuesta adaptada y contextualizada de la versión reducida del instrumento validado "Love Attitudes Style" (Hendrick et al. 1998).

Se llevó a cabo un muestreo no probabilístico accidental, muy común en este tipo de investigaciones (Bisquerra 2004; Grande y Abascal 2005; McMillan y Schumacher 2005), que dio lugar a una muestra final de 447 sujetos matriculados en la Universidad de Sevilla durante el curso 2013 (ver Tabla I). Aunque como índica el tipo de muestreo empleado, optamos por seleccionar a los sujetos basándonos en criterios de proximidad o accesibilidad, y no en el azar (muestreo probabilístico), tratamos de suavizar la consecuente falta de validez externa recurriendo a un tamaño muestral considerable y a la representatividad proporcional en cuanto al sexo y el área de conocimiento (Arte y Humanidades, Ciencias, Ingeniería y Arquitectura, Ciencias de la Salud, y Ciencias Sociales y Jurídicas).

Los participantes fueron, en su mayoría, alumnos menores de 23 años (81 \%) que se encontraban matriculados en alguno de los tres primeros cursos (75 $\%)$. Para el cálculo del tamaño muestral se tomaron como referencia los 57.965 alumnos matriculados en la Universidad de Sevilla durante el curso 2011-2012. En el caso de que hubiésemos podido elegir los sujetos al azar, con esos 447 participantes habríamos garantizado la representatividad muestral para un nivel de confianza del $95 \%$, un error del $+4,62 \%$ y $p=q$.

\section{CONSTRUCCIÓN Y VALIDACIÓN DEL INSTRUMENTO DE MEDIDA}

Entre el cuarto trimestre de 2012 y el primer bimestre de 2013 se realizó la traducción al español de la escala LAS y se procedió, mediante la técnica Delphi (Hung et al. 2008), a la validación de su formato y contenido. El panel estuvo formado por cuatro profesores de la Universidad de Sevilla, entre los que se encontraban filólogos en lengua inglesa, expertos en género y especialistas en metodología de la investigación, junto con nueve alumnas internas colaboradoras en esta investigación. En marzo de 2013 tuvo lugar una prueba piloto con 58 estudiantes, gracias a la cual obtuvimos una versión definitiva, a la que denominamos 'Escala ReLAS' (Revisión de la Escala Love Attitude Scale), que aglutinó las seis dimensiones tradicionales de estilos de amor (Eros, Ludus, Storge, Manía, Pragma y Ágape) en 22 ítems con 6 opciones de respuesta cada uno (0- Totalmente en desacuerdo, 1- Bastante en desacuerdo, 2- En desacuerdo, 3- De acuerdo, 4- Bastante de acuerdo, 5- Totalmente de acuerdo). En ella se eliminaron los ítems con cargas factoriales considerables en más de un factor y los que no saturaban convenientemente en ninguno de ellos. La recogida de los datos se efectuó en el segundo trimestre de 2013 y cumplió escrupulosamente con la legislación sobre protección de datos española (Ley orgánica 15/1999 de 13 de diciembre).

\section{ANÁlisis DE LOS DATOS}

Se realizó un análisis psicométrico, descriptivo e inferencial. La fiabilidad del instrumento se determinó mediante la prueba Alfa de Cronbach y la validez

Tabla I.

Características de la muestra

\begin{tabular}{|l|l|c|c|}
\hline \multicolumn{2}{|l|}{} & Recuento & $\%$ \\
\hline \multirow{4}{*}{ Sexo } & Hombre & 180 & $40,3 \%$ \\
\cline { 2 - 4 } & Mujer & 267 & $59,7 \%$ \\
\hline \multirow{4}{*}{ Estudios (Área de conocimiento) } & Arte y Humanidades & 55 & $12,3 \%$ \\
\cline { 2 - 4 } & Ciencias & 28 & $6,3 \%$ \\
\cline { 2 - 4 } & Ingeniería y Arquitectura & 132 & $29,6 \%$ \\
\cline { 2 - 4 } & Ciencias de la Salud & 48 & $10,8 \%$ \\
\cline { 2 - 4 } & Ciencias Sociales y Jurídicas & 183 & $41,0 \%$ \\
\hline \multirow{4}{*}{ Curso } & Primero & 55 & $12,3 \%$ \\
\cline { 2 - 4 } & Segundo & 127 & $28,5 \%$ \\
\cline { 2 - 4 } & Tercero & 155 & $34,8 \%$ \\
\cline { 2 - 4 } & Cuarto & 81 & $18,2 \%$ \\
\cline { 2 - 4 } & Quinto & 23 & $5,2 \%$ \\
\cline { 2 - 4 } & Postgrado & 5 & $1,1 \%$ \\
\hline \multirow{2}{*}{ Edad } & Media & \multicolumn{2}{|c|}{ Desviación T } \\
\cline { 2 - 4 } & 21,63 & 2,65 \\
\hline
\end{tabular}


de constructo a través del Análisis Factorial Exploratorio (AFE) por componentes principales. Esta técnica se aplicó tras haber descartado previamente, mediante la prueba KMO (Kaiser, Meyer y Olkin) y el test de esfericidad de Bartlett, que las correlaciones entre los ítems constituyesen una matriz de identidad, lo que desaconsejaría totalmente el uso de análisis factoriales. Para la rotación de los componentes, dada la existencia de correlaciones entre algunos de los factores, se utilizó el método Promax, que ayudó considerablemente a minimizar la cantidad de factores y el número de variables con cargas altas dentro de cada uno de ellos. Únicamente se aceptaron valores $R>0,4$ (Floyd \& Widaman 1995).

Para estimar la validez discriminante del instrumento se analizó si la correlación entre las variables latentes resultaba inferior a la unidad.

Para el análisis de los datos referentes a los estilos de amor se recurrió a porcentajes, medias y desviaciones típicas, mientras que para el estudio de diferencias en función del sexo empleamos la prueba $\mathrm{U}$ de Mann-Whitney (al no existir distribución normal de las puntuaciones) y la "d" de Cohen (1988).

\section{Resultados}

En cuanto a la validez de constructo de la escala ReLAS, los resultados de la prueba $\operatorname{KMO}(, 712)$ y del test de esfericidad de Bartlett $(p=, 000)$ muestran la pertinencia de desarrollar el AFE. Tras la aplicación de este, y tomando como criterio autovalores superiores a 1 , se corroboran las seis dimensiones (Eros, Ludus, Storge, Pragma, Manía y Ágape) del instrumento original con un poder explicativo de la varianza del $55,92 \%$. En la rotación Promax los ítems han obtenido pesos estadísticamente significativos $(p<, 01)$, entre, 414 y, 808 en el factor al que pertenecían (ver Tabla II), por lo que se estima que la validez de constructo de la estructura factorial podría considerarse aceptable. Con un número menor de factores, el porcentaje de varianza explicada resulta demasiado bajo $(51,12 \%)$. Obviamente, si en la retención mantenemos más de seis de los factores, el porcentaje de varianza explicado aumentaría, pero, al igual que con menos de seis, además de distanciarnos del número de dimensiones validadas en el instrumento original, obtendríamos una estructura difícil de explicar, tomando como referencia la literatura existente.

En cuanto a la fiabilidad del instrumento, se alcanzan valores alfa de Cronbach aceptables para cada una de las dimensiones (ver Tabla III), tomando como referencia los criterios de George y Mallery (2003).

Como podemos observar en la Tabla IV, las correlaciones entre los factores alcanzan valores bajos, situándose entre ,014 (correlación entre las subescalas Manía y Ludus) y ,449 (correlación en- tre Ágape y Manía). La inexistencia de correlaciones próximas a la unidad, nos hace descartar que dos factores representen en realidad una misma dimensión, hecho que avala la validez discriminante de la escala ReLAS, que presentaría dimensiones suficientemente diferenciadas.

En lo que respecta a los estilos de amor, tomando como referencia las puntuaciones medias globales alcanzadas en la escala ReLAS (ver Figura 1), podríamos concluir que los más extendidos serían, por orden, Ágape $(3,23)$, Eros $(2,86)$ y Storge $(2,77)$, quedando relegados a un segundo plano los de tipo Manía $(1,97)$, Pragma $(1,78)$ y Ludus $(1,77)$. Observando las medias globales para cada sexo, no parece que existan grandes diferencias entre hombres $y$ mujeres (ver Figura 1), si bien, algunas de ellas llegan a resultar estadísticamente significativas, como explicaremos más adelante.

Observando las medias de la Tabla $V$ podríamos afirmar, en términos generales, que el perfil de las relaciones entre los estudiantes de la Universidad de Sevilla se caracteriza por ayudar en tiempos de crisis a una pareja estable, con la que se tiene una "química especial", de la que han llegado a enamorarse tras un proceso de encariñamiento previo y a la que se quiere incondicionalmente pese a enfados ocasionales, lo que supone un acuerdo casi total con el ideal de amor romántico.

Adentrándonos en un análisis pormenorizado de las conductas que definen los distintos tipos de amor, observaríamos, refiriéndonos al estilo Ágape (ítems del 19 al 22), que más del $90 \%(91,6 \%)$ de los encuestados manifiesta tratar de ayudar a su pareja si está atravesando un momento complicado, llegando incluso, 6 de cada 10 (61,7\%), a manifestar que compartirían con su pareja todo lo que tienen, y prácticamente la mitad (51\%), a que sacrificarían sus propios deseos para cumplir los de su pareja. Tanto es así que 3 de cada 4 (74,4\%) afirman que seguirían queriendo incondicionalmente a su pareja, aunque se enfadasen.

En cuanto a Eros (ítems 1 y 2), un alto porcentaje $(88,3 \%)$ opina que es importante tener química con su pareja, sin embargo, el $64 \%(\bar{x}=1,90)$ afirma que su relación no se produjo por un "flechazo".

En lo que respecta al estilo Storge (ítems 4, 6 y 7 ), de amor basado en la amistad y la complicidad, el $78 \%$ manifiesta la necesidad de encariñarse con la pareja antes de llegar a enamorarse y prácticamente la mitad $(49,9 \%)$, que sus relaciones amorosas han surgido tras una buena amistad que esperan mantener aunque su relación fracasase (55,5\%).

En cuanto al tipo de amor Manía, obsesivo, celoso, con fuerte dependencia de la pareja (ítems 15 al 18), registramos que prácticamente 7 de cada 10 encuestados $(67,5 \%)$ se angustia si sospecha que su pareja puede estar con otra persona, y casi la mi- 
Tabla II.

Matriz de configuración

\begin{tabular}{|c|c|c|c|c|c|c|}
\hline \multirow{2}{*}{ ÍTEMS } & \multicolumn{6}{|c|}{ Componente } \\
\hline & 1 & 2 & 3 & 4 & 5 & 6 \\
\hline $\begin{array}{l}\text { 1. En cuanto nos conocimos fue amor a primera vista. Parece que estábamos } \\
\text { predestinados el uno para el otro. }\end{array}$ & 105, &,- 092 & 174 & 210 &,- 385 & ,445 \\
\hline 2. Tenemos química. Me gusta y me parece guap@. &,- 146 & ,264 & ,228 & ,166 &,- 082 & ,488 \\
\hline 3. Cuando nos conocimos tuvimos relaciones sexuales muy pronto. & 047 &,- 016 &,- 027 &, 579 &,- 256 & 151 \\
\hline 4. Para enamorarme, necesito encariñarme antes con esa persona. & ,119 & 057 &,- 021 & ,122 & ,414 & 640 \\
\hline 5. Puedo superar los temas de desamores con bastante facilidad y rapidez. & ,177 & 027 &,- 417 &, 500 & 028 &,- 105 \\
\hline 6. Mis relaciones amorosas más satisfactorias han partido de buenas amistades. &,- 074 & ,087 & ,245 &,- 029 & ,768 & ,128 \\
\hline $\begin{array}{l}\text { 7. Espero ser siempre amigo de la persona que quiero. Mantengo buena amistad } \\
\text { con casi todas las personas con las que he tenido una relación. }\end{array}$ & , 105 & , 196 &,- 039 & ,103 &, 563 & ,042 \\
\hline $\begin{array}{l}\text { 8. Cuando mi pareja se vuelve demasiado dependiente, prefiero echar marcha } \\
\text { atrás. Prefiero estar un poco insegur@ acerca de mi compromiso. }\end{array}$ & ,225 &,- 213 & 119 & ,410 & ,292 & ,041 \\
\hline 9. A veces he tenido dos amantes sin que ningun@ de I@s dos lo supiese. &,- 181 &,- 108 & 123 & ,655 & 072 &,- 112 \\
\hline 10.Me gusta "enrollarme" (tener sexo), con un $n^{\circ}$ de compañer@s diferentes. &,- 140 &,- 012 &,- 109 & ,698 & 079 &,- 067 \\
\hline $\begin{array}{l}\text { 11. Antes de comprometerme, tengo en cuenta lo que esa persona va a llegar a ser } \\
\text { en la vida. Lo mejor es tener una pareja con una formación similar. }\end{array}$ & ,741 & ,037 &,- 124 &,- 060 & ,086 &, 114 \\
\hline $\begin{array}{l}\text { 12. Trato de planificar mi vida bien, y tengo en cuenta cómo afectará a mi carrera } \\
\text { profesional antes de elegir una pareja. }\end{array}$ & ,786 &,- 080 &,- 047 &,- 014 & 075 & ,087 \\
\hline 13.Al elegir pareja tengo en cuenta cómo le va a caer a mi familia. & ,767 & 014 &, 212 &,- 114 &,- 120 &,- 106 \\
\hline 14.Al elegir pareja tengo en cuenta si será un buen padre/madre. & ,676 & 105 &, 237 &, 006 &,- 065 &,- 078 \\
\hline $\begin{array}{l}\text { 15.Cuando me enamoro, a veces me siento tan inquiet@ que tengo problemas para } \\
\text { concentrarme en otra cosa o poder dormir. }\end{array}$ & 119, &,- 234 & ,762 &,- 139 & 085 &,- 083 \\
\hline 16. Me angustio si sospecho que mi pareja está con otra persona. & ,088 &,- 029 &, 578 & 087 &,- 070 & ,290 \\
\hline 17. Si mi pareja me ignora, a veces hago cosas tontas para atraer su atención. &,- 008 &, 006 &, 657 &,- 025 & ,236 &,- 093 \\
\hline $\begin{array}{l}\text { 18. Cuando he tenido un fracaso sentimental, me siento tan deprimid@ que hasta } \\
\text { he pensado en el suicidio }\end{array}$ &,- 084 & 197 &, 552 & ,139 & ,209 &,- 346 \\
\hline 19. Trato de ayudar siempre a mi pareja en épocas difíciles. &,- 102 &, 598 & 197 &,- 182 & 005 & 099 \\
\hline 20.Prefiero sacrificar mis propios deseos para que mi pareja logre los suyos. & 157 & ,734 &,- 155 &,- 142 & 106 &,- 133 \\
\hline 21. Todo lo que tengo es para compartirlo con mi pareja. &,- 002 & ,703 &, 034 &,- 044 &, 136 & 157 \\
\hline $\begin{array}{l}\text { 22. Aunque nos enfadáramos, seguiría queriendo total e incondicionalmente a mi } \\
\text { pareja. }\end{array}$ &,- 094 & ,808 &,- 176 &,- 074 & ,147 & ,022 \\
\hline
\end{tabular}

Nota: Método de extracción: Análisis de componentes principales. Explica el 55,92 \% del porcentaje total de varianza explicada.

Método de rotación: Normalización Promax con Kaiser.

La rotación converge en 14 iteraciones.

Tabla III.

Fiabilidad del instrumento de medida.

\begin{tabular}{|l|c|c|}
\hline Dimensiones & Ítems & Fiabilidad \\
\hline Ágape & 19 al 22 & .715 \\
\hline Eros & 1,2 & .676 \\
\hline Storge & 4,6 y 7 & .670 \\
\hline Manía & 15 al 18 & .684 \\
\hline Pragma & 11 al 14 & .763 \\
\hline Ludus & $3,5,8$ al 10 & .793 \\
\hline
\end{tabular}


Tabla IV.

Matriz de correlaciones de componentes.

\begin{tabular}{|c|c|c|c|c|c|c|}
\hline Componente & Pragma & Ágape & Manía & Ludus & Storge & Eros \\
\hline Pragma & 1,000 &, 045 &, 136 &, 032 &, 225 &,- 141 \\
\hline Ágape &, 045 & 1,000 &, 449 &,- 047 &,- 100 &, 089 \\
\hline Manía &, 136 &, 449 & 1,000 &, 014 &,- 147 &, 046 \\
\hline Ludus &, 032 &,- 047 &, 014 & 1,000 &,- 041 &,- 057 \\
\hline Storge &, 225 &,- 100 &,- 147 &,- 041 & 1,000 &,- 268 \\
\hline Eros &,- 141 &, 089 &, 046 &,- 057 &,- 268 & 1,000 \\
\hline
\end{tabular}

Figura 1.

Puntuaciones medias globales.

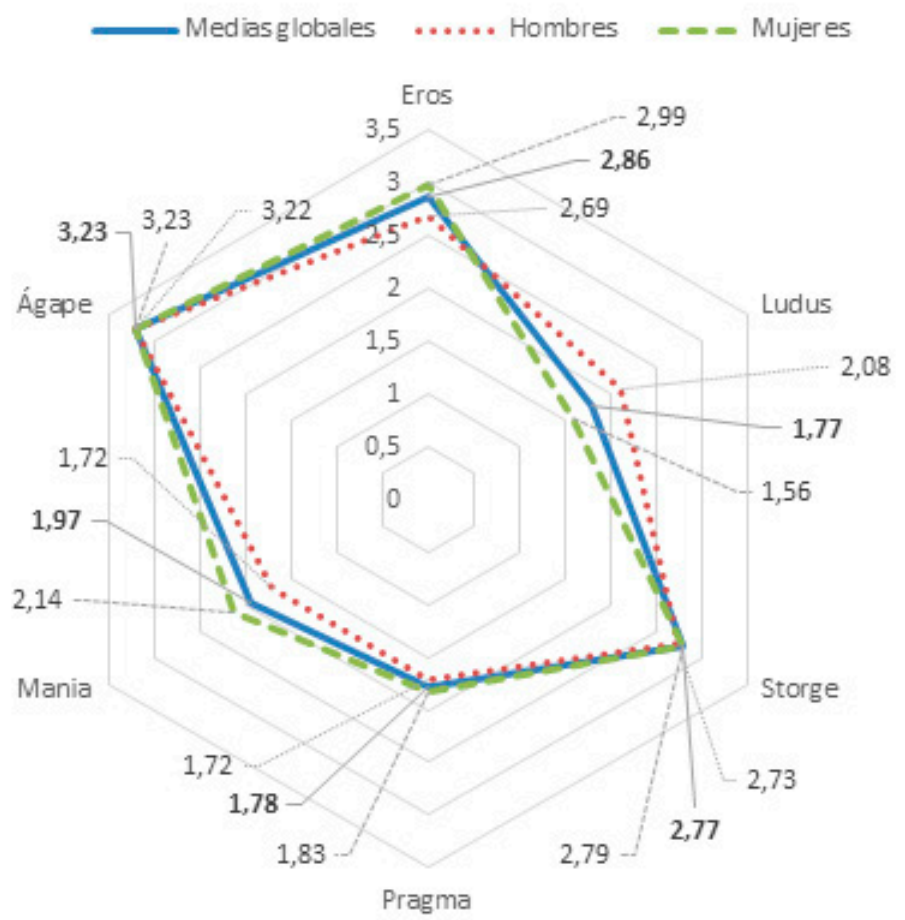

Tabla V.

Opiniones del alumnado con respecto a las conductas que definen los tipos de amor.

\begin{tabular}{|l|c|c|c|c|c|c|c|c|}
\hline \multicolumn{1}{|c|}{ Ítems de la escala RELAS } & $\mathbf{0}$ & $\mathbf{1}$ & $\mathbf{2}$ & $\mathbf{3}$ & $\mathbf{4}$ & $\mathbf{5}$ & $\overline{\mathbf{x}}$ & $\mathbf{\delta}$ \\
\hline $\begin{array}{l}\text { 1. En cuanto nos conocimos fue amor a } \\
\text { primera vista. Parece que estábamos } \\
\text { predestinados el uno para el otro. }\end{array}$ & $26,8 \%$ & $16,8 \%$ & $20,4 \%$ & $19,3 \%$ & $8,8 \%$ & $7,9 \%$ & 1,90 & 1,58 \\
\hline $\begin{array}{l}\text { 2. Tenemos química. Me gusta y me } \\
\text { parece guap@. }\end{array}$ & $1,8 \%$ & $2,3 \%$ & $7,7 \%$ & $20,8 \%$ & $35,4 \%$ & $32,1 \%$ & 3,82 & 1,14 \\
\hline $\begin{array}{l}\text { 3. Cuando nos conocimos tuvimos } \\
\text { relaciones sexuales muy pronto. }\end{array}$ & $18,1 \%$ & $12,7 \%$ & $20,9 \%$ & $18,8 \%$ & $14,7 \%$ & $14,7 \%$ & 2,44 & 1,67 \\
\hline $\begin{array}{l}\text { 4. Para enamorarme, necesito } \\
\text { encariñarme antes con esa persona. }\end{array}$ & $5,0 \%$ & $7,3 \%$ & $9,6 \%$ & $23,3 \%$ & $26,8 \%$ & $27,9 \%$ & 3,43 & 1,43 \\
\hline $\begin{array}{l}\text { 5. Puedo superar los temas de } \\
\text { desamores con bastante facilidad y } \\
\text { rapidez. }\end{array}$ & $16,7 \%$ & $20,2 \%$ & $24,5 \%$ & $21,3 \%$ & $9,9 \%$ & $7,3 \%$ & 2,09 & 1,46 \\
\hline $\begin{array}{l}\text { 6. Mis relaciones amorosas más } \\
\text { satisfactorias han partido de buenas } \\
\text { amistades. }\end{array}$ & $21,2 \%$ & $15,5 \%$ & $13,4 \%$ & $19,4 \%$ & $18,5 \%$ & $12,1 \%$ & 2,35 & 1,71 \\
\hline $\begin{array}{l}\text { 7. Espero ser siempre amigo de la persona } \\
\text { que quiero. Mantengo buena amistad } \\
\text { con casi todas las personas con las que } \\
\text { he tenido una relación. }\end{array}$ & $17,0 \%$ & $14,0 \%$ & $14,5 \%$ & $24,2 \%$ & $14,9 \%$ & $15,4 \%$ & 2,52 & 1,67 \\
\hline
\end{tabular}




\begin{tabular}{|c|c|c|c|c|c|c|c|c|}
\hline $\begin{array}{l}\text { 8. Cuando mi pareja se vuelve } \\
\text { demasiado dependiente, prefiero } \\
\text { echar marcha atrás. Prefiero estar } \\
\text { un poco insegur@ acerca de mi } \\
\text { compromiso. }\end{array}$ & $25,2 \%$ & $18,4 \%$ & $18,2 \%$ & $17,7 \%$ & $15,5 \%$ & $5,0 \%$ & 1,95 & 1,56 \\
\hline $\begin{array}{l}\text { 9. A veces he tenido dos amantes sin } \\
\text { que ningun@ de I@s dos lo supiese. }\end{array}$ & $59,7 \%$ & $12,6 \%$ & $6,2 \%$ & $8,5 \%$ & $5,0 \%$ & $8,0 \%$ & 1,11 & 1,65 \\
\hline $\begin{array}{l}\text { 10.Me gusta "enrollarme" (tener sexo), } \\
\text { con un } n^{\circ} \text { de compañer@s diferentes. }\end{array}$ & $52,9 \%$ & $12,9 \%$ & $9,0 \%$ & $10,9 \%$ & $7,7 \%$ & $6,6 \%$ & 1,27 & 1,65 \\
\hline $\begin{array}{l}\text { 11. Antes de comprometerme, tengo en } \\
\text { cuenta lo que esa persona va a llegar } \\
\text { a ser en la vida. Lo mejor es tener una } \\
\text { pareja con una formación similar. }\end{array}$ & $27,2 \%$ & $15,4 \%$ & $16,3 \%$ & $19,0 \%$ & $14,3 \%$ & $7,7 \%$ & 2,01 & 1,64 \\
\hline $\begin{array}{l}\text { 12. Trato de planificar mi vida bien, y } \\
\text { tengo en cuenta cómo afectará a mi } \\
\text { carrera profesional antes de elegir una } \\
\text { pareja. }\end{array}$ & $29,2 \%$ & $15,4 \%$ & $18,3 \%$ & $18,1 \%$ & $10,9 \%$ & $8,1 \%$ & 1,90 & 1,63 \\
\hline $\begin{array}{l}\text { 13. Al elegir pareja tengo en cuenta cómo } \\
\text { le va a caer a mi familia. }\end{array}$ & $35,3 \%$ & $17,9 \%$ & $16,3 \%$ & $15,2 \%$ & $11,1 \%$ & $4,3 \%$ & 1,62 & 1,56 \\
\hline $\begin{array}{l}\text { 14.Al elegir pareja tengo en cuenta si } \\
\text { será un buen padre/madre. }\end{array}$ & $34,3 \%$ & $20,9 \%$ & $13,9 \%$ & $16,8 \%$ & $9,5 \%$ & $4,5 \%$ & 1,60 & 1,54 \\
\hline $\begin{array}{l}\text { 15. Cuando me enamoro, a veces me siento } \\
\text { tan inquiet@ que tengo problemas para } \\
\text { concentrarme en otra cosa o poder } \\
\text { dormir. }\end{array}$ & $19,5 \%$ & $16,6 \%$ & $15,6 \%$ & $23,1 \%$ & $14,1 \%$ & $11,1 \%$ & 2,29 & 1,63 \\
\hline $\begin{array}{l}\text { 16. Me angustio si sospecho que mi pareja } \\
\text { está con otra persona. }\end{array}$ & $11,4 \%$ & $8,0 \%$ & $13,0 \%$ & $20,6 \%$ & $22,0 \%$ & $24,9 \%$ & 3,08 & 1,64 \\
\hline $\begin{array}{l}\text { 17. Si mi pareja me ignora, a veces hago } \\
\text { cosas tontas para atraer su atención. }\end{array}$ & $23,5 \%$ & $20,4 \%$ & $18,1 \%$ & $18,6 \%$ & $11,8 \%$ & $7,7 \%$ & 1,98 & 1,58 \\
\hline $\begin{array}{l}\text { 18. Cuando he tenido un fracaso } \\
\text { sentimental, me siento tan deprimid@ } \\
\text { que hasta he pensado en el suicidio. }\end{array}$ & $77,3 \%$ & $8,8 \%$ & $4,8 \%$ & $3,6 \%$ & $3,2 \%$ & $2,3 \%$ &, 53 & 1,18 \\
\hline $\begin{array}{l}\text { 19. Trato de ayudar siempre a mi pareja } \\
\text { en épocas difíciles. }\end{array}$ & $4,1 \%$ & $1,1 \%$ & $3,2 \%$ & $9,8 \%$ & $25,9 \%$ & $55,9 \%$ & 4,20 & 1,23 \\
\hline $\begin{array}{l}\text { 20.Prefiero sacrificar mis propios deseos } \\
\text { para que mi pareja logre los suyos. }\end{array}$ & $9,2 \%$ & $15,4 \%$ & $24,4 \%$ & $27,9 \%$ & $15,7 \%$ & $7,4 \%$ & 2,47 & 1,37 \\
\hline $\begin{array}{l}\text { 21. Todo lo que tengo es para compartirlo } \\
\text { con mi pareja. }\end{array}$ & $5,5 \%$ & $12,8 \%$ & $20,0 \%$ & $24,8 \%$ & $23,5 \%$ & $13,4 \%$ & 2,88 & 1,40 \\
\hline $\begin{array}{l}\text { 22. Aunque nos enfadáramos, seguiría } \\
\text { queriendo total e incondicionalmente a } \\
\text { mi pareja. }\end{array}$ & $2,7 \%$ & $8,2 \%$ & $14,7 \%$ & $24,3 \%$ & $26,1 \%$ & $24,0 \%$ & 3,35 & 1,35 \\
\hline
\end{tabular}

tad $(48,1 \%)$ manifiesta tener problemas de concentración cuando está enamorado. Lo que resulta verdaderamente alarmante es que prácticamente 1 de cada 10 sujetos $(9,1 \%)$ haya manifestado, en mayor o menor medida, acuerdo con el planteamiento de suicidio tras un fracaso sentimental.

Parece ser que el interés, propio del amor Pragma (ítems del 11 al 14), no es una de las características más destacable entre los jóvenes universitarios. Son muchos los que afirman no haber fijado como criterios para elegir pareja la idoneidad como futuro padre o madre $(69,1 \%)$, o la facilidad para encajar en la familia (65,9\%). Por otro lado, el $63 \%$ manifiesta no haberse planteado cómo afectaría esa potencial pareja a su carrera profesional, aunque el $41 \%$ sí reconoce haber empleado como filtro lo que, supuestamente, llegaría a ser esa persona en la vida.

Los encuestados han mostrado un claro desacuerdo con las conductas Ludus (ítems 3, 5, 8, 9 y 10). El $78,5 \%$ manifiesta no haber contado con más de un amante al mismo tiempo sin que ninguno de ellos estuviese al tanto, llegando a desagradarle la idea de contar con distintas parejas sexuales al 74,8 $\%$. Algo más de la mitad $(51,7 \%)$ ni siquiera mantuvo relaciones hasta bastante tiempo después de haberse conocido. La mayoría no supera con facilidad los temas de desamor (61,8 \%), les cuesta superar rupturas y cambiar de pareja, aunque resulta llamativo el hecho de que 6 de cada 10 encuestados (61,8\%) prefieran frenar la relación cuando su pareja comienza a mostrar una dependencia emocional.

En general, la dispersión de las opiniones de los encuestados, como podemos comprobar al observar la desviación típica obtenida en los distintos ítems, ha sido relativamente reducida, registrándose valores entre 1,18 y 1,71. Los menores grados de acuerdo se han registrado en algunas conductas tipo Storge (ítem 6 y 7) y tipo Ludus (ítems 3, 9 y 10).

El estudio de diferencias en función del sexogénero continúa siendo hoy día una práctica habitual en estudios de diversa índole (Montañés et al. 2015). Como podemos apreciar en la Tabla VI, se ha constatado la existencia diferencias significativas en algunas de las conductas valoradas. Los hombres 
manifiestan en mayor medida superar con facilidad y rapidez los problemas de desamor, tener diferentes parejas sexuales y dar marcha atrás en la relación cuando la otra persona se vuelve demasiado dependiente de ellos. Todas ellas son conductas que representarían un amor tipo Ludus. En dos de ellas (facilidad para superar el desamor y promiscuidad), el nivel de diferencia registrado se consideraría moderado (d ronda los, 5 puntos) y en la tercera (dar marcha atrás en la relación), pequeño (Cohen 1988). Por el contrario, las mujeres presentan niveles significativamente superiores en algunas conductas de los estilos Eros, Pragma, Ágape y Manía, como tener química con su pareja, elegirla tomando en consideración cómo va a encajar en la familia, tener problemas de concentración cuando se enamoran, angustiarse si sospechan infidelidad, llamar la atención cuando esta las ignora y tratar de ayudarla en momentos difíciles. Atendiendo a los valores de la columna d, todas estas diferencias habría que considerarlas pequeñas, tomando como referencia los criterios de Cohen (1988). No se han encontrado diferencias significativas en ninguna de las conductas que definen el estilo Storge.

\section{Discusión Y CONCLUSIONES}

En un primer análisis global, los estilos de amor más aceptados por ellos y ellas son similares. A idéntica conclusión llegan $\mathrm{C}$. Hendrick y $\mathrm{S}$. Hendrick (1986) tras emplear la escala LAS en una muestra de 807 estudiantes de la Universidad de Miami, y Bosch et al. (2013), tras emplear la escala LAS para analizar los estilos de amor en una muestra de 1351 personas de diferentes edades de la población española. Estudios como el de Fehr, Harasymchuk y Sprecher (2014), en el ámbito universitario americano, tampoco han encontrado diferencias por género en cuanto los estilos de amor.
La tendencia de la muestra es hacia el estilo Ágape, el amor altruista y de renuncia, que considera la relación como una negación del individualismo en un proceso de abnegación, entrega y sacrificio, por el otro, para 'toda la vida'. Consideramos que este responde a los parámetros culturales actuales de amor romántico, el amor altruista y de renuncia (Esteban y Távora 2008; Ferrer et al. 2010). Estos estereotipos saturan toda la producción mediática que socializa en el romance desde la infancia con metamensajes como "lo daría todo por amor", "sin ti no soy nada", "el amor lo puede todo", etc. y van creando toda una mitología relacionada con el amor romántico. Estos resultados coinciden con nuevos hallazgos en las parejas universitarias (Fehr, Harasymchuk y Sprecher 2014), que afirman valores elevados de altruismo, cuidado y solidaridad hacia la pareja, propios del estilo Ágape. Este estilo estaría relacionado con comportamientos de ayuda desinteresada, cuidados y valores prosociales. Probablemente estas relaciones, donde hay mayor compromiso y apoyo en la pareja, sean más satisfactorias y estables, pero no podemos confirmar que estas afirmaciones se correspondan absolutamente con la realidad, ya que habría que comprobar la veracidad de estos autoinformes evaluando acciones concretas, no percepciones.

En nuestro estudio, Ludus es el estilo de menor aceptación, coincidiendo con otras investigaciones en estudiantes universitarios estadounidenses (C. Hendrick y S. Hendrick 1986; Hendrick et al. 1988; S. Hendrick, C. Hendrick 1995), mexicanos (Valdez, González y Sánchez 2005), japoneses (Kanemasa et al. 2004), argentinos (Brenlla et al. 2001; Liporace 2001) y brasileños (Andrade y García 2009). A Idénticas conclusiones apuntan también los estudios que han tomado como muestra distintos segmentos de la

Tabla VI.

Conductas significativamente diferentes en hombres y mujeres (U de Mann-Whitney).

\begin{tabular}{|c|c|c|c|c|}
\hline \multirow{2}{*}{ Ítems de la escala ReLAS } & \multicolumn{2}{|c|}{ Rango. Promedio. } & \multirow[b]{2}{*}{ Sig. } & \multirow[b]{2}{*}{ d } \\
\hline & Hombre & Mujer & & \\
\hline 2. Tenemos química. Me gusta y me parece guap@. & 202,05 & 235,52 &, 005 & ,26 \\
\hline 5. Puedo superar los temas de desamores con bastante facilidad y rapidez. & 252,70 & 195,35 &, 000 & ,48 \\
\hline $\begin{array}{l}\text { 8. Cuando mi pareja se vuelve demasiado dependiente, prefiero echar marcha atrás. Prefiero estar } \\
\text { un poco insegur@ acerca de mi compromiso. }\end{array}$ & 236,57 & 209,58 &, 026 & ,22 \\
\hline 10. Me gusta "enrollarme" (tener sexo), con un nº de compañer@s diferentes. & 268,33 & 189,63 &, 000 & 69 \\
\hline 13. Al elegir pareja tengo en cuenta cómo le va a caer a mi familia. & 203,37 & 233,84 &, 011 & ,26 \\
\hline $\begin{array}{l}\text { 15. Cuando me enamoro, a veces me siento tan inquiet@ que tengo problemas para concentrarme } \\
\text { en otra cosa o poder dormir. }\end{array}$ & 195,52 & 238,41 &, 000 &, 33 \\
\hline 16. Me angustio si sospecho que mi pareja está con otra persona. & 192,78 & 236,34 &, 000 & ,33 \\
\hline 17. Si mi pareja me ignora, a veces hago cosas tontas para atraer su atención. & 200,20 & 236,00 &, 003 & ,27 \\
\hline 19. Trato de ayudar siempre a mi pareja en épocas difíciles. & 192,98 & 239,20 &, 000 & ,30 \\
\hline
\end{tabular}

Nota: El estudio del tamaño del efecto se ha desarrollado atendiendo a la fórmula de Cohen (1988), considerándose para los cálculos desviaciones típicas combinadas. 
población general, como los de Yela (2012) o Ferrer et al. (2008), en el contexto español, o los de Neto (2012) en Portugal, quien investigó a individuos de 18 a 90 años para poder demostrar que aunque la sexualidad es un aspecto importantísimo de las relaciones, queda relegado a un segundo plano tras el amor romántico.

Las diferencias significativas encontradas en función del sexo podríamos relacionarlas con ciertos postulados sociobiológicos de la perspectiva evolucionista que vincula el amor y el sexo (Simpson y Kenrick 1997), y también con perspectivas culturalistas que explican esto por la socialización diferencial de género que se produce desde la infancia, y que perpetúa las desigualdades entre mujeres y hombres (Ferrer y Bosch 2013). Estas predicen que los hombres tenderán a valorar más el amor lúdico, el sexo casual y la obtención del máximo número de parejas posibles, lo que resulta coherente con su menor inversión parental (Yela 1998; Buss et al. 1990), y que las mujeres, debido a la mayor inversión y esfuerzo parental, tenderán a valorar menos este estilo de amor. Estos resultados coinciden con los de otros estudios comparativos (C. Hendrick y S. Hendrick 1986; S. Hendrick y C. Hendrick 1995; Brenlla et al. 2001; Solano 2001; Díaz-Loving y Robles 2009; Bosch et al. 2013; Espinoza et al. 2014) que exponen como los chicos son significativamente más Ludus que las chicas. Las mujeres, comparadas con los hombres, son más conservadoras y rechazan una relación sexual inmediata, mientras que los hombres se muestran más permisivos con ese tipo de relaciones y son proclives a mantenerlas con parejas diferentes (C. Hendrick y S. Hendrick 1986; S. Hendrick y C. Hendrick 1995; Yela 2012). Puede ayudar a explicar ese dato el hecho de que la deseabilidad social actual de jóvenes y adolescentes responde a los principios de la llamada "contrarrevolución sexual", así como al temor a las enfermedades de transmisión sexual. Es perfectamente explicable desde la perspectiva antropológica y culturalista, desde donde nos situamos en este artículo, que sostiene la existencia de una socialización amorosa diferencial por géneros.

En cuanto al estilo Pragma en nuestra muestra universitaria, las chicas preparan su futuro académico y profesional sin buscar un compañero que le aporte necesariamente seguridad económica y social (León y Mora 2010). Recordemos además que al estudiar las diferencias significativas en función del sexo, solo mostraban un poco más de preocupación que los chicos en lo concerniente a valorar cómo iba a encajar la pareja en su familia. Estos datos contradicen, en parte, los resultados de otras investigaciones (Brenlla et al. 2001; Ubillos et al. 2001; S. Hendrick y C. Hendrick 2002; Ferrer et al. 2008) que vienen a resaltar la tendencia de la mujer a evaluar a sus parejas masculinas a partir de su capacidad económica, poder social, o status, y la de los hombres a valorar más la belleza, la juventud y características relacionadas con el amor lúdico. Históricamente, las mujeres han recibido una socialización donde el sexo es considerado como "un valor que debe ser vigilado" y reservado para un marido proveedor de recursos (Hendrick et al. 1988; Yela 2003). Nuestros resultados demuestran que las universitarias no buscan el estatus a través de sus relaciones afectivas.

Se confirman pues las tesis sobre el cambio de parámetros que se ha producido en las expectativas sobre el amor y la pareja (Illouz 2012). Los cambios sociales de las últimas décadas han disminuido la brecha de género y hacen que la experiencia amorosa sea distinta, ya no se exige a la pareja, en lo individual, el rol de proveedor de recursos y estatus social que se valoraba antes. Por tanto, las mujeres, sobre todo las universitarias, no buscan este elemento en la pareja. Pero también podríamos hacer una lectura desde las claves evolucionistas de Helen Fisher (2005; 2014): "estamos volviendo al pasado"; en la mayoría de las sociedades la mujer ha entrado en el mercado laboral, tiene mayor acceso a la educación y al poder socioeconómico, por tanto, se vuelve a recuperar el rol evolutivo de la mujer recolectora de hace millones de años, con un estatus y cotas de poder similares a las del hombre. Esto concuerda también con las conclusiones de Valdez et al. (2008), obtenidas al comparar las diferencias en la elección de pareja que se establecen entre la cultura argentina y la mexicana. Todo ello indica que la evolución de la mujer en las últimas décadas en nuestro país, en cuanto a la formación universitaria y participación en el mundo laboral se refiere, ha supuesto disminuir las desigualdades en las expectativas de la vida privada (Ajenjo y García 2014). Recordemos además que al estudiar las diferencias significativas en función del sexo, solo mostraban un poco más de preocupación que los chicos en lo concerniente a valorar cómo iba a encajar la pareja en su familia. Lo cual resulta comprensible, pues nuestra muestra corresponde a Sevilla, una ciudad de Andalucía donde el valor de la familia tradicional es todavía un anclaje de peso para los individuos.

En lo referente al estilo Manía, 'el amor de los síntomas', aunque nuestros resultados, al igual que el de otros estudios (Sánchez-Aragón 2009a), reflejan significativamente cómo las mujeres están socializadas en la centralidad del amor y lo viven de forma más posesiva y celosa que los hombres, lo cierto es que los resultados a nivel global son relativamente bajos. De hecho es uno de los tipos de amor menos predominante entre los estudiantes de la Universidad de Sevilla. Distintos estudios (S. Hendrick y C. Hendrick 1995; Sánchez-Aragón 2009b) vienen a señalar que este estilo de amor es más característico en los adolescentes y, según Retana y Sánchez-Aragón 
(2005), se irá transformando en Ludus a medida que madure el individuo. Kanemasa et al. (2004), en sus investigaciones sobre la población universitaria adolescente (edad media de 19 años) corrobora este hecho al situar en primer lugar al estilo Manía, seguido por Eros, Ágape, Ludus y Storge. Sin embargo, Ubillos et al. (2001), con su investigación en 15 países y diferentes grupos étnicos, obtiene que el estilo Eros es el más valorado, seguido de Storge y Manía. C. Hendrick y S. Hendrick (1986) ofrecen también interesantes diferencias en las concepciones sobre el amor en función de la cultura que se analice. Exponen cómo los estudiantes orientales tienen puntuaciones relativamente bajas en Eros, y altas en Storge y Pragma, mientras que los hispanos tienden más al Ludus que los no hispanos. Señalan cómo las culturas que enfatizan el amor centrado en la amistad y el compañerismo valoran también la practicidad y otorgan escasa importancia a la posesión, los celos $y$, en general, a la carga emocional intensa.

Nuestra investigación, tomando como referencia el estudio de García y Cedillo (2011) sobre el tipo de publicaciones que ha suscitado la temática del amor, ha seguido pautas muy similares al resto de trabajos realizados en esta línea. Como limitaciones a destacar sería interesante recoger nuevos datos que nos permitiesen comprobar si ese alejamiento del estilo Pragma, individualista y mercantilista, propio de sociedades capitalistas, es real, o si por el contrario, como afirma Illouz (2009), tienden a él sin ni tan siquiera ser conscientes de ello, razón por la que, según esta autora, existe esa discrepancia entro lo que afirman y la realidad. Por otro lado, consideramos que también sería interesante efectuar un contraste por áreas de conocimiento. Para ello, hemos de ampliar el tamaño muestral en cada una de ellas e incluir representantes de todos los centros universitarios que engloba cada área. Estamos convencidos de que los diferentes rasgos de la personalidad, unidos a aspectos culturales de diversa índole, influyen en los estudios que se seleccionan y que encontraremos un patrón de asociación entre estos y los tipos de amor. Por otra parte, aunque el análisis psicométrico de escala ReLAS es aceptable, sería necesario, además de efectuar un análisis de tipo confirmatorio, trabajar con nuevas muestras para mejorar los parámetros de la misma. Sería interesante también estudiar la relación que guardan los tipos de amor con la violencia de género, asociación ya apuntada en otros estudios (Garrido 2001; González y Santana 2001; Sanmartín et al. 2003; Ferrer y Bosch 2013), con objeto de estimar la validez predictiva de la escala ReLAS en la detección de potenciales agresores.

En conclusión, y tomando como referencia los resultados obtenidos, parece ser que la forma de entender el amor de pareja que subyace en nuestra muestra universitaria está relacionada con un concepto idealizado y romántico del amor. Es un amor Ágape, altruista y abnegado, en el que la sexualidad y la sensualidad son aspectos secundarios. Esta idea prevalece sobre los aspectos más pasionales, visuales o de atracción analizados en Eros, pero con gran implicación en los aspectos colaborativos y amistosos propios del estilo Storge. Hay un claro rechazo por parte de chicos y chicas hacia las relaciones sexuales esporádicas o sin implicación emocional, pese a que se registren diferencias significativas que indican conductas más celosas y posesivas en las mujeres y relaciones de índole más sexual en los chicos. Tomando como referencia únicamente sus propias apreciaciones, no parece que busquen en la pareja un vehículo de ascenso en el estatus económico o social, quizás porque se trata de una muestra universitaria donde las chicas están apostando por un futuro académico y profesional.

A pesar de los avances de la mujer en estas últimas décadas, la incorporación de esta a la universidad y su sobreformación universitaria respecto a los varones, consideramos que la ideología que subyace en el amor romántico vuelve a ser la trampa de sujeción del patriarcado moderno.

\section{REFERENCIAS BibLIOGRÁfiCAS}

Adrián, J. A. 2007. "Hacia una fenomenología de los afectos: Heidegger y Max Scheler". Thémata. Revista de Filosofía 39: 365-368.

Ajenjo, M. y J. García. 2014. "Cambios en el uso del tiempo de las parejas. ¿Estamos en el camino hacia una mayor igualdad?" Revista Internacional de Sociología 72: 453-476. http://dx.doi.org/10.3989/ris.2012.05.28

Andrade, L. y A. García. 2009. "Atitudes e crenças sobre o amor: Versão brasileira da escala de Estilos de Amor". Interpersona: An International Journal on Personal Relationships 3 (1): 89-102. https://doi.org/10.5964/ijpr. v3i1.34
Barbosa, S., J. Ayala, B. Peña, O. Rangel y A. Ortiz. 2011. "Relación entre el tipo de apoyo y el estilo de amor en parejas". Enseñanza e Investigación en Psicología 16: 41-56.

Barrón, A., D. Martínez-Iñigo, P. de Paul and C. Yela. 1999. "Romantic beliefs and myths in Spain". The Spanish Journal of Psychology 2: 64-73.

Bisquerra, R. 2004. Metodología de la investigación educativa. Madrid: La Muralla.

Brenlla, M. E., A. Brizzio y A. Carrera. 2001. "Actitudes hacia el amor y apego". Psicodebate. Psicología, Cultura y Sociedad 4: 7-23. 
Bosch, E., V. Ferrer, V. Ferreiro y C. Navarro. 2013. La violencia contra las mujeres. El amor como coartada. Barcelona: Anthropos.

Buss, D. M. et al. 1990. "International preferences in selecting mates: A study of 37 cultures". Journal of Cross Cultural Psychology 21: 5-47. http://dx.doi. org/10.1177/0022022190211001

Castells, M. y M. Subirats. 2007. Mujeres y hombres. ¿Un amor imposible? Madrid: Alianza Editorial.

Cohen, J. 1988. Statistical Power Analysis for the Behavioral Sciences. Hillsdale, N. J.: Erlbaum.

Díaz-Loving, R. y S. Robles. 2009. "Atracción, romance, sexo y protección en adolescentes". Enseñanza e Investigación en Psicología 14: 215-228.

Eagly, A. H. and W. Wood. 1999. "The origins of sex differences in human behavior". American Psychologist 54: 404423. http://dx.doi.org/10.1037/0003-066X.54.6.408

Engels, F. [1884] 2008. El origen de la familia, la propiedad privada y el Estado. Madrid: Alianza Editorial.

Espinoza, A. V., F. E. Correa y L. F. García. 2014. "Percepción social de la infidelidad y estilos de amor en la pareja". Enseñanza e Investigación en Psicología 19: 135-147.

Esteban M. L. y A. Távora. 2008. "El amor romántico y la subordinación social de las mujeres: revisiones y propuestas". Anuario de Psicología 39: 59-73.

Fehr, B., C. Harasymchuk and S. Sprecher. 2014. "Compassionate love in romantic relationships. A review and some new findings". Journal of Social and Personal Relationship 31: 601-629. http://dx.doi. org/10.1177/0265407514533768

Ferrer, V. A., E. Bosch, C. Navarro, M. C. Ramis y E. García. 2008. "El concepto de amor en España". Psicothema 20: 589-595.

Ferrer, V. A., E. Bosch y C. Navarro, 2010. "Los mitos románticos en España”. Boletín de Psicología 99: 7-31.

Ferrer, V. y E. Bosch, 2013. "Del amor romántico a la violencia de género. Para una coeducación emocional en la agenda educativa". Profesorado 17: 105-122.

Fisher, H. E. 1992. Anatomy of Love: The Natural History of Monogamy, Adultery, and Divorce. New York: W. W. Norton.

Fisher, H. E. 2005. Por qué amamos: naturaleza y química del amor romántico. Madrid: Suma de Letras.

Fisher, H. E. 2014. "The Tyranny of Love". Pp. 237-265 in Behavioral Addictions: Criteria, Evidence and Treatment. http://dx.doi.org/10.1016/b978-0-12-407724-9.00010-0

Floyd, F. J. and K. F. Widaman. 1995. "Factor analysis in the development and refinement of clinical assessment instruments". Psychological Assessment 7: 286-299. http://dx.doi.org/10.1037/1040-3590.7.3.286

García, A. y R. P. Cedillo. 2011. "La normalización científica del amor. A propósito de la perspectiva evolutiva en psicología". Revista Latinoamericana de Estudios sobre Cuerpos, Emociones y Sociedad 6: 83-95.

Garrido, V. 2001. Amores que matan. Acoso y violencia contra las mujeres. Valencia: Algar.

González, R. y J. D. Santana. 2001. Violencia en parejas jóvenes. Análisis y prevención. Madrid: Pirámide.

George, D. and P. Mallery. 2003. SPSS for Windows step by step: A simple guide and reference. 11.0 update. Boston: Allyn and Bacon.

Grande, I. y E. Abascal. 2005. Análisis de encuestas. Madrid: ESIC.
Hendrick, C. and S. Hendrick, 1986. "A Theory and Method of Love". Journal of Personality and Social Psychology 50: 392-402. http://dx.doi.org/10.1037/00223514.50.2.392

Hendrick, S., C. Hendrick and N. Adler. 1988. "Romantic Relationships: Love, Satisfaction, and Staying Together". Journal of Personality and Social Psychology 54: 980988. http://dx.doi.org/10.1037/0022-3514.54.6.980

Hendrick, S. and C. Hendrick. 1995. "Gender differences and similarities in sex and love". Personal Relationships 2: 55-65. http://dx.doi.org/10.1111/j.1475-6811.1995. tb00077.x

Hendrick, C., S. Hendrick and A. Dicke. 1998. "The Love Attitudes Scale: Short form". Journal of Personal and Social Relationships 15: 147-159. http://dx.doi. org/10.1177/0265407598152001

Hendrick, S. and C. Hendrick. 2002. "Linking romantic love with sex: Development of the Perceptions of Love and Sex Scale". Journal of Social and Personal Relationships 19: 361-378. http://dx.doi. org/10.1177/0265407502193004

Hung, H. L., J. W. Altschuld and Y. Lee. 2008. "Methodological and conceptual issues confronting a cross-country Delphi study of educational program evaluation". Evaluation and Program Planning 31: 191-198. http:// dx.doi.org/10.1016/j.evalprogplan.2008.02.005

Illouz, E. 2007. Intimidades congeladas. Las emociones en el capitalismo. Madrid: Katz.

Illouz, E. 2009. El consumo de la utopía romántica. El amor y las contradicciones culturales del capitalismo. MadridBuenos Aires: Katz.

Illouz, E. 2012. Por qué duele el amor. Una explicación sociológica. Buenos Aires: Katz.

Jónasdóttir, A. 1993. El poder del amor. ¿Le importa el sexo a la democracia?. Madrid: Cátedra.

Kanemasa, Y., J. Taniguchi. I. Daibo and M. Eishimori. 2004. "Love styles and romantic love Experiences in Japan". Social Behavior and Personality 32: 265-282. http:// dx.doi.org/10.2224/sbp.2004.32.3.265

Lee, J. A. 1973. Colours of love: An exploration of the ways of loving. Toronto: New Press.

León, F. J. y E. Mora. 2010. "Género y vocación científica. Un estudio de caso basado en mecanismos". Revista Internacional de Sociología 68: 399-428. http://dx.doi. org/10.3989/ris.2008.06.19

Liporace, M. F. 2001. "Autodescripciones sexuales y estereotipos de género en estudiantes terciarios y universitarios". Psicodebate. Psicología, Cultura y Sociedad 4: 89-104.

McMillan, J. H. y S. Schumacher. 2005. Investigación educativa. Madrid: Pearson Addison Wesley.

Montañés, P., J. L. Megías, S. de Lemus y M. Moya. 2015. "Sexismo en la adolescencia: influencia de las primeras relaciones de pareja". Revista de Psicología Social 30: 219-240. http://dx.doi.org/10.1080/21711976.2 015.1016756

Moreno, M. y G. Sastre. 2010. Cómo construimos universos. Amor, cooperación y conflicto. Barcelona: Gedisa.

Navarro, L. 2006. "Modelos ideales de familia en la sociedad española". Revista Internacional de Sociología 43: 119-138.

Neto, F. 2012. "Perceptions of love and sex across the adult life span". Journal of Social and Personal Relationships 29: 760-775. http://dx.doi.org/10.1177/0265407512443638 
Padilla, N. y R. Díaz-Loving. 2013. "Premisas familiares y socioculturales del emparejamiento. Enseñanza e Investigación en Psicología 18: 249-262.

Petersen, J. and C. Thompson. 2005. "Perceptual Differences Between Adults and Adolescents on Meeting Their Need for Love". International Journal of Reality Therapy 25: 1-17.

Retana, B. E. y R. Sánchez-Aragón. 2005. "Construcción y validación de una escala para medir adicción al amor en adolescentes". Enseñanza e Investigación en Psicología 10: 127-141.

Rodríguez-Castro, Y., M. Lameiras-Fernández, M. V. Carrera-Fernández y P. Vallejo-Medina. 2013. "La fiabilidad y validez de la escala de mitos hacia el amor: las creencias de los y las adolescentes". Revista de Psicología Social 28: 157-168. http://dx.doi. org/10.1174/021347413806196708

Rubin, G. S. 2011. Deviations: A Gale Rubin Reader. Durham, NC: Duke University Press. https://www.dukeupress. edu/deviations

Sánchez-Aragón, R. 2009a. "Efectos diferenciales del bienestar subjetivo, autorrealización y celos en las fases del amor pasional". Enseñanza e Investigación en Psicología 14: 5-21.

Sánchez-Aragón, R. 2009b. "Expectativas, percepción de estabilidad y estrategias de mantenimiento en las relaciones amorosas". Enseñanza e Investigación en Psicología 14: 229-243.

Sangrador, J. 1993. "Consideraciones psicosociales sobre el amor romántico". Psicothema 5: 181-196.

Sanmartín, J., A. Molina e Y. García. 2003. Informe internacional 2003. Violencia contra la mujer en las relaciones de pareja. Estadísticas y legislación. Valencia: Centro Reina Sofía para el Estudio de la Violencia.

Scheler, M. 1996. Ordo Amoris. Madrid: Caparrós editores, S.L.
Simpson, J. A. and D. T. Kenrick. 1997. Evolutionary Social Psychology. Mahwah: Lawrence Erlbaum Associat.

Solano, A. C. 2001. "¿Qué entienden jóvenes y adultos por 'estar enamorado'?” Psicodebate. Psicología, Cultura y Sociedad 4: 25-38.

Sternberg, R. 1986. "A Triangular Theory of Love". Psychological Review93: 119-135. http://dx.doi.org/10.1037/0033295X.93.2.119

Sternberg, R. 1996. "Historias de amor". Psicología Contemporánea 3: 4-17.

Ubillos, S., E. Zubieta, D. Páez, J. C. Deschamps, A. Ezeiza y A. Vera. 2001. "Amor, cultura y sexo". Revista Electrónica de Motivación y Emoción 4: 8-9. Consulta el 4 de septiembre 2016 (http://reme.uji.es/articulos/aubils9251701102/texto.html).

Valdez, J. L., N. I. González y Z. P. Sánchez. 2005. "Elección de pareja en universitarios mexicanos". Enseñanza e Investigación en Psicología 10: 355-367.

Valdez, J. L., N. I. González, J. Arce, S. González, G. Morelato y M. S. Ison. 2008. "La elección de pareja real e ideal en dos culturas: México y Argentina. Un análisis por sexo". Enseñanza e Investigación en Psicología 13: 261-277.

Yela, C. 1998. "Diferencias entre sexos en los juicios sobre su comportamiento amoroso y sexual". Revista de Psicología General y Aplicada 51: 115-147.

Yela, C. 2003. "La otra cara del amor: mitos, paradojas y problemas". Encuentros en Psicología Social 1: 263-267.

Yela, C. 2012. "Análisis de la Teoría de las Estrategias Sexuales en la población española". Psicothema 24: 48-54.

Walker, S. and L. Barton. 1988. Gender, class and education. Nueva York: Falmer Press.

JAVIER RODRÍGUEZ-SANTERO. Profesor Titular de Universidad en el Departamento de Métodos de Investigación y Diagnóstico en Educación (MIDE) de la Facultad de Educación de la Universidad de Sevilla. Su trayectoria investigadora se centra en los métodos de investigación, el análisis de datos y la evaluación orientada al aprendizaje. En los últimos años ha participado en distintos proyectos y contratos de investigación relacionados con esas temáticas y ha ejercido como evaluador de manuscritos para revistas científicas de impacto. Miembro del grupo de Investigación PAIDI SEJ-467.

Ma ÁNGELES GARCÍA-CARPINTERO MUÑOZ. Profesora Titular de Universidad. Licenciada en Antropología Social y Cultural, Profesora del Departamento de Enfermería de la Universidad de Sevilla. Especializada en influencia del Género en la Salud, ha participado en diversos proyectos y publicaciones relacionados con la violencia de género y el amor romántico. Miembro del grupo de Investigación PAIDI CTS969: Innovación en Cuidados y Determinantes Sociales en Salud.

ANA MARÍA PORCEL GÁLVEZ. Doctorada en Enfermería. Profesora Contratada Doctora. Directora del Departamento de Enfermería de la Universidad de Sevilla. Profesora vinculada a la línea Procedimientos, Intervenciones y Resultados en Salud, Programa de Doctorado en Ciencias de la Salud, Universidad de Sevilla. Evaluadora e Investigadora Principal en Proyectos I+D+I Consejería de Salud, Junta de Andalucía. Vocal grupo gestor PAIDI CTS969: Innovación en Cuidados y Determinantes Sociales en Salud. 